\title{
LARGE EDDY SIMULATION OF FLOW THROUGH ARTIFICIAL MACRO-POROUS STRUCTURE
}

\author{
T. Kořínek ${ }^{*}$, T. Tisovskýy ${ }^{* *}$, J. Hujer ${ }^{* * *}$
}

\begin{abstract}
Numerical study of flow through artificial macro-porous structure is presented. The Large Eddy Simulation (LES) approach with dynamic sub-grid scale model were performed using opensource softwareOpenFOAM. The porous structure is created using an array of blocks. The effect of porosity of the artificial structure on turbulence properties is investigated.
\end{abstract}

Keywords: computational fluid dynamics, turbulent flow, large eddy simulation, openFOAM, porous structure

\section{Introduction}

The motivation of the study is to further understand of the flow through a macro-porous structure in order to simulate in detail the adsorption process on activated carbon (Kořínek, 2017). The aim of this study is to point out an influence of porous structure on turbulence properties. Due to difficulties in creating sufficiently fine and well structured computational grid of real porous structure, a creation of an artificial porous structure is commonly used method for reducing the computational cost and for further understand the flow behavior (Jungreuthmayer et al., 2015). In porous structures and microchannels with holes as small as a mean free path of molecule, the continuum hypothesis is not valid and molecular dynamics simulation methods like the Direct Simulation Monte Carlo are used rather than the Finite Volume Method applied on the Navier Stokes equations (Tisovský, 2017). A crucial parameter is the Knudsen number which compares the molecular mean free path with a characteristic length scale.

$$
K_{n}=\frac{\lambda}{L},
$$

where $\lambda$ is the mean free path of molecules and $\mathrm{L}$ is characteristic length scale. It has to be mentioned that this study was a complex problem, where macro-porous and micro-porous structure were incorporated into one domain. The Knudsen number for the macro-porous region was $K_{n}=1.8 \cdot 10^{-8}$ and $K_{n}=0.029$ for micro-porous region.

\section{Porous structure}

The porous structure was represented by array of simple blocks each representing micro-porous element. In order to simplify the problem, only flow through the macro-porous structure was modelled and the microporous structure was represented by a source of pressure drop. Approach used is significantly different from the approach traditionally used for modelling flow through porous structures (Zhang et al., 2017; Jungreuthmayer et al., 2015). In this case, number of structures with different dimensions of blocks and width of the slot between blocks were created. An overview of tested cases is shown in Tab. 1.

Ing. Tomáš Kořínek: Department of Power Engineering Equipment, Technical University of Liberec, Studentská 1402/2; 461 17, Liberec; CZ, tomas.korinek@tul.cz

** Ing. Tomáś Tisovský: Department of Power Engineering Equipment, Technical University of Liberec, Studentská 1402/2; 461 17, Liberec; CZ, tomas.tisovsky@tul.cz

*** Ing. Jan Hujer: Department of Power Engineering Equipment, Technical University of Liberec, Studentská 1402/2; 461 17, Liberec; CZ, jan.hujer@tul.cz 
Tab. 1: Case study

\begin{tabular}{|c|c|c|}
\hline case & size of the micro-porous block ( $\mathrm{mm} \times \mathrm{mm})$ & width of the slot (mm) \\
\hline B1 & $5 \times 5$ & 5 \\
\hline $\mathrm{B} 2$ & $5 \times 3.7$ & 2.5 \\
\hline B3 & $2.5 \times 2.5$ & 2.5 \\
\hline B4 & $2.5 \times 2.5$ & 1.25 \\
\hline
\end{tabular}

Fig. 1: Artificial porous structures B2 (left) and B4 (right)

The dimension of the channel containing macro-porous structure were as follows: height $=10 \mathrm{~mm}$, width $=$ $25 \mathrm{~mm}$ and length $=100 \mathrm{~mm}$. The artificial structure created is shown in Fig. 1, where a light gray color corresponds to the fluid and a dark gray color is related to the micro-porous structure.

\section{Numerical strategy}

The fully 3D numerical simulation using the Large Eddy Simulation (LES) approach was performed to obtain velocity and pressure field. The filtered unsteady governing equations for incompressible fluid flow are:

$$
\frac{\partial \bar{u}_{i}}{\partial t}+\frac{\partial \bar{u}_{i} \bar{u}_{j}}{\partial x_{j}}=-\frac{1}{\rho} \frac{\partial \bar{p}}{\partial x_{i}}+\frac{\partial}{\partial x_{j}}\left[\left(\nu+\nu_{s g s}\right) \frac{\partial \bar{u}_{i}}{\partial x_{j}}\right]+R_{i},
$$

where $\mathrm{p}$ is pressure, $\mathrm{R}$ is pressure loss of micro-porous block based on Darcy-Forchheimer law, $\mathrm{u}$ is velocity, $\nu$ is kinematic viscosity, $\nu_{s g s}$ is sub-grid scale eddy viscosity, $\rho$ is density. And transport equation for subgrid scale kinetic energy is:

$$
\frac{\partial k_{s g s}}{\partial t}+\frac{\partial \bar{u}_{i} k_{s g s}}{\partial x_{i}}=2 \nu_{s g s} \bar{S}_{i j}+C_{\epsilon} \frac{k_{s g s}^{3 / 2}}{\Delta}+\frac{\partial}{\partial x_{j}}\left(\nu_{s g s} \frac{\partial k_{s g s}}{\partial x_{j}}\right),
$$

where model constant $C_{\epsilon}=1.048, k_{s g s}$ is subgrid scale kinetic energy, $S_{i j}$ is strain rate tensor, $\Delta$ is the cubic root of the volume of computational cell. The first term on right side represents the production of sub-grid scale kinetic energy, the second term is dissipation and the third term is diffusion. The sub-grid scale eddy viscosity $\nu_{t}$ is defined as:

$$
\nu_{s g s}=C_{k} \Delta \sqrt{k_{s g s}}
$$

where model constant $C_{k}=0.094$. Due the problem with incorrect behaviour of $\nu_{s g s}$ near wall, the van Driest damping function was used.

A divergence free synthetic eddy method introduced by (Jarrin et al., 2009) and improved by (Poletto et al., 2013) was used to initialise turbulence on inlet. The bulk velocity at inlet was $5 \mathrm{~m} / \mathrm{s}$. The Crank Nicolson 
scheme was chosen for temporal discretization for its second order accuracy. The PISO algorithm was used to solve the governing equations. Tolerance of residuals was set to $10^{-7}$ for pressure and $10^{-6}$ for velocity and subgrid scale kinetic energy. Time step size was chosen based on the Courant Friedrich Lewy condition (CFL) with value of $\mathrm{CFL}=0.1$ for an initialization of the simulation and for the averaging part, the time step size was chosen based on the mean time step size from the initialization.

Four computational meshes were generated in order to perform mesh independence study with various number of cells for each mesh (673 920, 1073 250, 1500 000, 2171 650). However the coarsest mesh still satisfied the condition $y^{+}$at the wall should be around 1 . The mesh study was performed on the case B1 and results from the mesh study were used for creation of computational grids for cases B2 (2 964 000), B3 (8 370 000) and B4 (16 773 480).

\section{Results}

An influence of porous structure on vortices is shown in Fig. 2 using an iso-surface of Q-criterion. The iso-surfaces are both for the same value of normalized Q-criterion. The upper image is for the structure B1 and the lower image is for the structure B3. Smaller vortex structures are generated for the structure B3, which is more detailed in comparison with structure B1. It has been noted by (Bošković-Vragolivić et al., 2013) that with increase of bulk velocity, mass transfer to the structure is also increasing. Also the more detailed structure has a twice bigger surface of blocks, which leads to greater mass transfer to these blocks. However greater blockage results in increase in pressure loss.

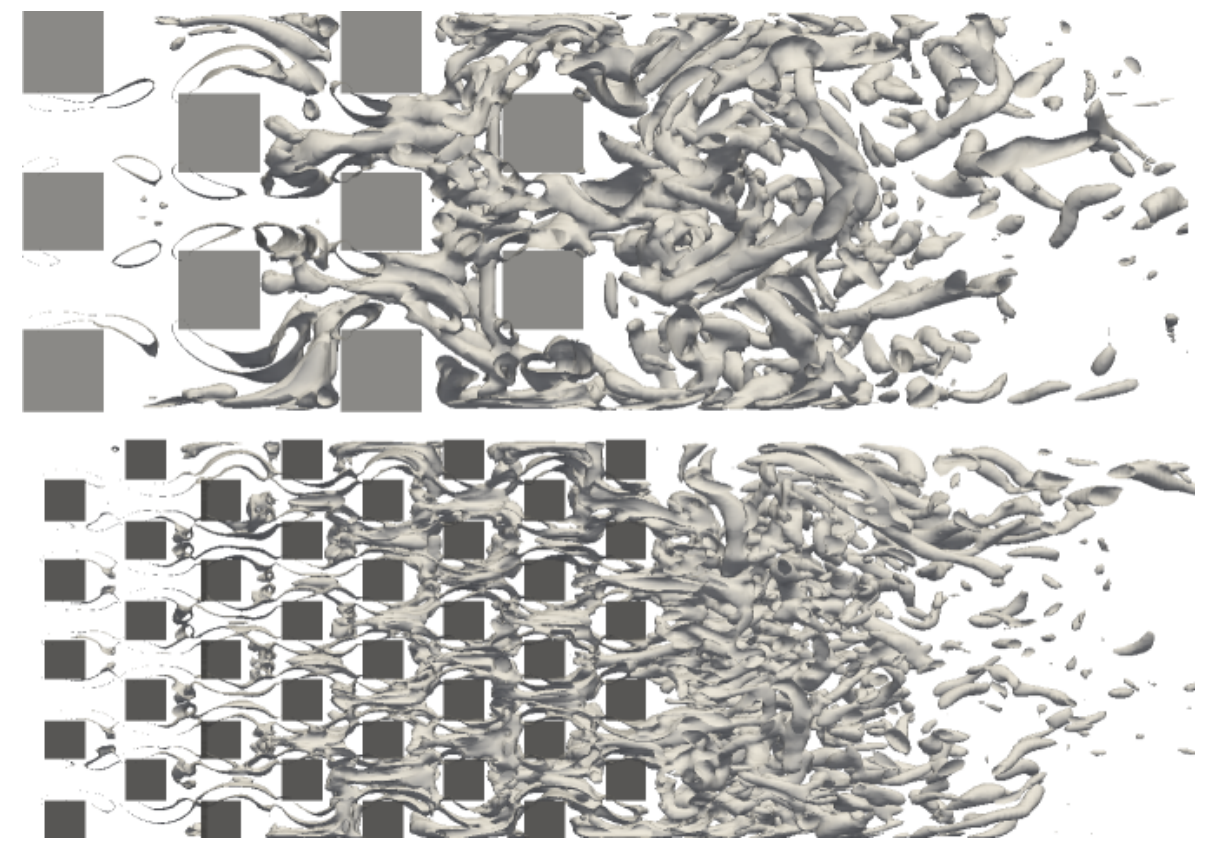

Fig. 2: Instantaneous iso-surface of Q-criterion for artificial structure B1 (up) and B3 (down).

It has to be pointed out that not only mean velocity has an influence on mass transfer, there is also influence of fluctuating part of velocity on intensity of mass transfer to the structure. Tab. 1 shows turbulence intensity for various artificial structures. A very interesting phenomenon is occurring for situation of different dimensions of block and slot. If the dimensions differ, the turbulence intensity is greater than for case where dimensions are same.

Tab. 2: An influence of structure on turbulence intensity

\begin{tabular}{lcccc}
\hline Structure & B1 & B2 & B3 & B4 \\
\hline Turbulence intensity (\%) & 10.2 & 28.1 & 9.48 & 26.4 \\
\hline
\end{tabular}




\section{Conclusions}

The macro-porous artificial structure with micro-porous elements were numerically investigated by LES. Various structures were compared to each other in order to evaluate critical parameters which have influence on mass transfer to the micro-porous elements.

Turbulence intensity was more influence by the ratio of the block width and the width of the slot, than the size of the block itself. If the size of the block and the size of the slot were same, turbulence intensity was lower than for case where dimensions differ. Simulations were performed for isothermal flow. Simulations of a non-isothermal flow will be carried out in the future.

\section{Acknowledgments}

This publication was written at the Technical University of Liberec with the support of the Specific University Research Grant No. 21227, as provided by the Ministry of Education, Youth and Sports of the Czech Republic in the year 2018. Computational resources were provided by the CESNET LM2015042 and the CERIT Scientific Cloud LM2015085, provided under the programme "Projects of Large Research, Development, and Innovations Infrastructures".

\section{References}

Bošković-Vragolivić, N., Garić-Grulović, R., Pjanović, R. and Grbavčić, Ž. (2013) Mass transfer and fluid flow visualization for single cylinder by the adsorption method. Int. J. Heat and Mass Transfer, Vol 59, pp 155-160.

Davidson, L. and Peng, S. (2013) Embedded Large-Eddy Simulation Using the Partially Averaged Navier-Stokes Model. AIAA Journal, Vol 51(5), pp 1066-1079.

Gnatowska, R. (2011) Aerodynamic Characteristics of Three-Dimensional Surface-Mounted Objects in Tandem Arrangement. International Journal of Turbo and Jet Engines, Vol 28, pp 21-29.

Jarrin, N., Prosser, R.,Uribe, J.-C., Benhamadouche, S. and Laurence, D. (2009) Reconstruction of turbulent fluctuations for hybrid RANS/LES simulations using a synthetic-eddy method. Int. J. Heat Fluid Flow, Vol 30, pp $435-442$.

Jungreuthmayer, CH., Steppert, P., Sekot, G., Zankel, A., Reingruber, H., Zanghellini, J. and Jungbauer, A. (2015) The 3D pore structure and fluid dynamics simulation of macroporous monoliths: High permeability due to alternating channel width. J. of Chromatography A, Vol 1425, pp 141-149.

Kořínek, T. (2017) An adsorption of carbon dioxide on activated carbon controlled by temperature swing adsorption. AIP Conference Proceedings, Vol 1889, pp 1-5.

Piller, M., Casagrande, D., Schena, G. and Santini, M. (2014) Pore-scale simulation of laminar flow through porous media. Journal of Physics: Conference Series, Vol 501, pp 1-15.

Poletto, R.,Craft, T. and Revell, A. (2013) A New Divergence Free Synthetic Eddy Method for the Reproduction of Inlet Flow Conditions for LES. Flow Turbulence Combust, Vol 91, pp 519-539.

Tisovský, T. (2017) Direct simulation Monte Carlo method for gas flows in micro-channels with bends with added curvature. EPJ Web of Conferences, Vol 143, pp 141-149.

Zhang, Z., Cheng, J. and He, X. (2017) Numerical simulation of flow and heat transfer in composite PCM on the basis of two different models of open-cell metal foam skeletons Int. J. Heat Mass Transfer, Vol 112, pp 959-971. 